The International Journal of Engineering and Science (IJES)

|| Volume || 6 || Issue || 4 || Pages || PP 56-59 || 2017 ||

ISSN (e): 2319 - 1813 ISSN (p): $2319-1805$

THE IJES

\title{
Development Of Industrial Automatic Multi Colour Sorting and Counting Machine Using Arduino Nano Microcontroller and TCS3200 Colour Sensor
}

\author{
Amitesha Sachdeva ${ }^{1}$, Mahesh Gupta ${ }^{2}$, Manish Pandey ${ }^{3}$, Prabham Khandelwal ${ }^{4}$ \\ Department of Mechanical Engineering SRM University, Modinagar Ghaziabad, India.
}

\begin{abstract}
-
Sorting of the products in the industry is very difficult task and continuous manual sorting creates issues. It is very desirable to create a machine that identify the objects and relocate them if the object meets certain criteria. This paper presents a solution to sort the coloured objects with the help of the robotic arm. The objects when placed on the conveyor belt are sorted based on colour sensing and are relocated to specific location. When an object moves from one location to another on the conveyor belt, the sensors give the input to the microcontroller which then give the command to the robotic arm to do the task. TCS3200 colour sensor is used for detection of the colour of the object. DC motors are used to move the conveyor belt, gripper and the lifter. Arduino Nano microcontroller is used to give the commands. L293D motor driver is used to drive the motors and LCD display makes the system user friendly.
\end{abstract}

Keywords-Colour sorting, Conveyor belt, DC motor, LCD display, L293D motor driver, Microcontroller, Robotic arm, TCS3200 colour sensor.

Date of Submission: 14 April 2017

Date of Accepted: 03 May 2017

\section{INTRODUCTION}

Nowadays, the competition is so intense that the efficiency of the product is regarded as the key to success. The efficiency of the product includes the speed of the production, lowering material and labour cost, improving quality and decreasing the rejection. Taking all the things under consideration this project is developed which is very useful for industries.

The aim of this project is to obtain fully automatic material handling system. This is done with the help of microcontroller unit. This unit synchronizes the movement of the robotic arm to pick the moving objects on the conveyor belt. It sorts the coloured object moving on the conveyor belt by picking and placing them in their respective preprogramed place. This leads to the elimination of the monotonous work done by human, achieving accuracy and speed in the production. This project involves colour sensors that senses the objects colour and sends signal to the microcontroller. Based on the detection of the colour, the robotic arm moves to the specific location and releases the object and comes back to original position. As the objects are been sorted, simultaneously the counting of the objects is also done.

By considering all this our project will have:

1. Sorting of object based on colour.

2. Counting the objects which were sorted, and display of count.

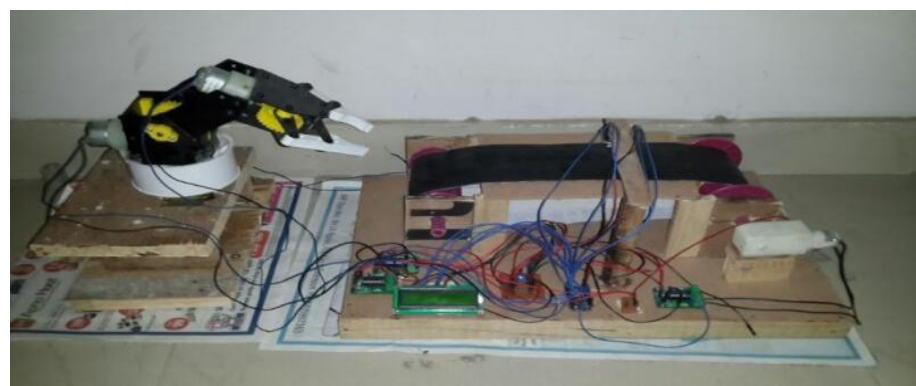

Figure 1: Colour Sorting Machine 


\section{Components}

\section{Conveyor Belt}

The conveyor belt $(30 \times 9 \mathrm{~cm})$ used here consists of two wheels on which the belt moves. There is the continuous loop of the material over the rotating wheels. One of the wheels called the driver wheel is powered by the motor and other unpowered wheel is called idler. This leads to the movement of the conveyor belt that is used to carry the objects over it.

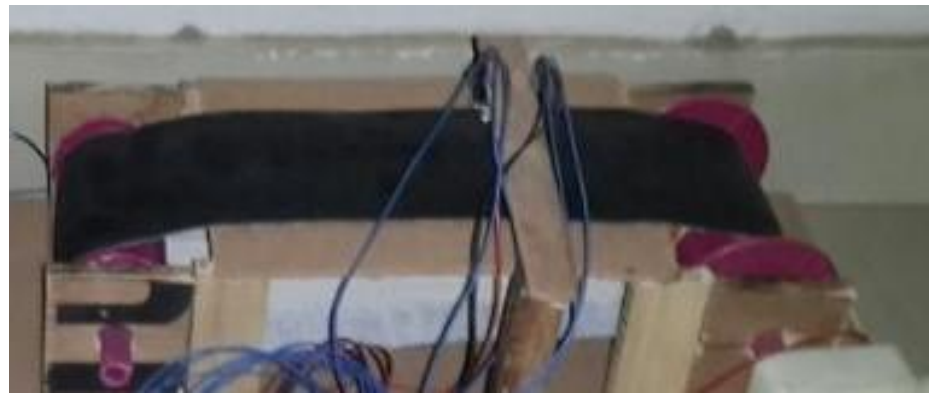

Figure 2: Conveyor Belt

\section{Gripper}

The DC motor is used to control the opening and closing movement of the gripper. The DC motor receives its signal from the microcontroller for performing the operations. The gripper ( 2 jaws) is designed specially for grabbing the objects running on the conveyor belt and dropping on specified locations.

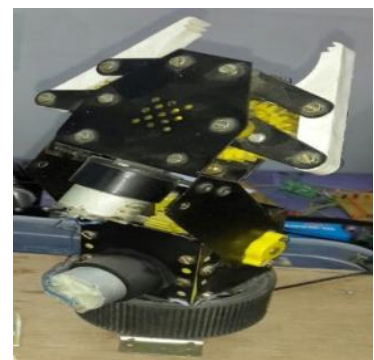

Figure 3: Robotic Gripper

\section{Colour Sensor}

The colour sensor used is TCS3200 Color Sensor which is a complete color detector, including a TAOS TCS3200 RGB sensor chip and 4 white LEDs. The TCS3200 can detect and measure a nearly limitless range of visible colors. The TCS3200 has an array of photo detectors, each with either a red, green, or blue filter, or no filter (clear). Internal to the device is an oscillator which produces a square-wave output whose frequency is proportional to the intensity of the chosen color.

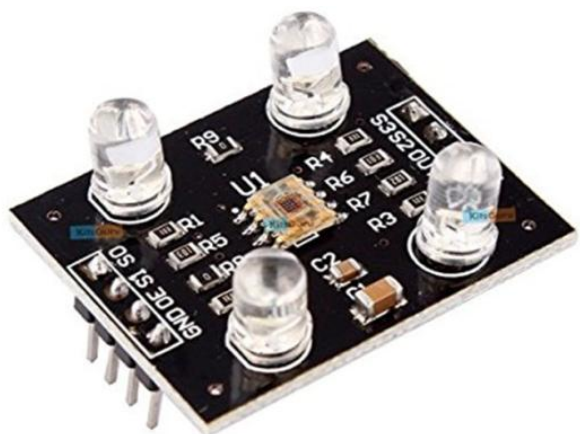

Figure 4: TCS3200 colour Sensor

\section{Microcontroller}

In this project microcontroller Arduino Nano (ATmega168) is used for motion control and object detection. The microcontroller works on the set of instructions that are preprogrammed and stored in the memory. It then takes the instructions from its program and one by one operates as the instructions and carries the required operations. 


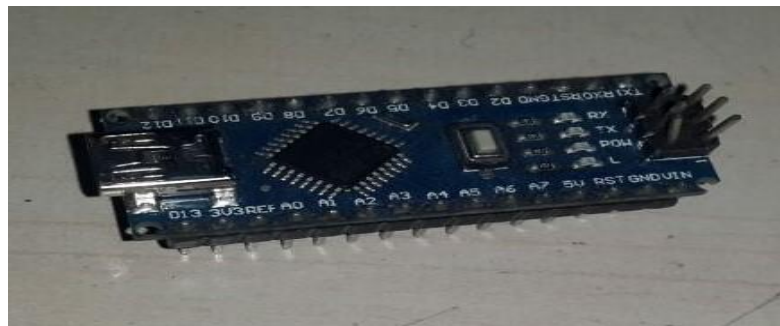

Figure 5: Microcontroller Arduino Nano

\section{Motor}

DC motor (6-12V at 1000rpm) is a device that convert electrical form of energy into mechanical form of energy. DC motor is powered by DC current. Our project has 3 high torque DC motors for driving. One motor drives the driver of the conveyor belt and other two are used for movement of robotic arm. L293D Motor driver is interfaced with microcontroller to control the robotic arm.

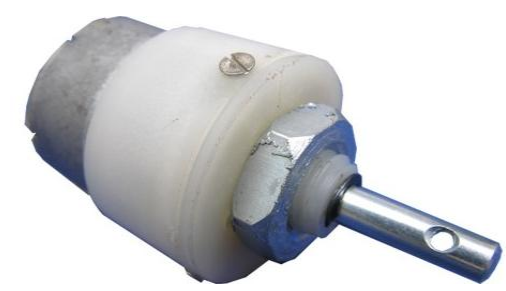

Figure 6: Geared DC Motor

\section{LCD Display}

A liquid-crystal display (LCD) is a flat panel display, electronic visual display, or video display that uses the light modulating properties of liquid crystals. We are using 16x2 LCD display which has 2 horizontal line comprising a space of 16 displaying character.

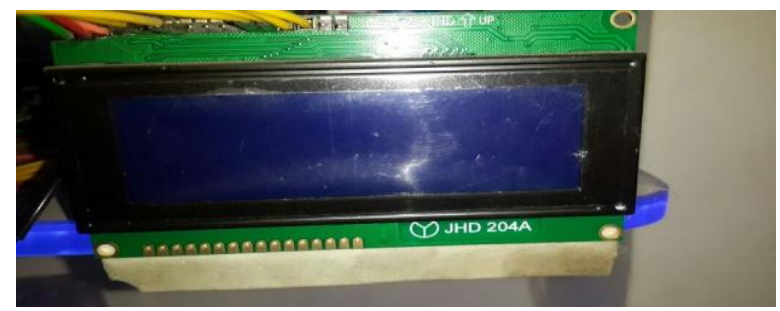

Figure 7: LCD Display

\section{Motor Driver}

L293D is a dual H-bridge motor driver integrated circuit (IC). Motor drivers act as current amplifiers since they take a low-current control signal and provide a higher-current signal. This higher current signal is used to drive the motors.L293D contains two inbuilt H-bridge driver circuits. In its common mode of operation, two DC motors can be driven simultaneously, both in forward and reverse direction.

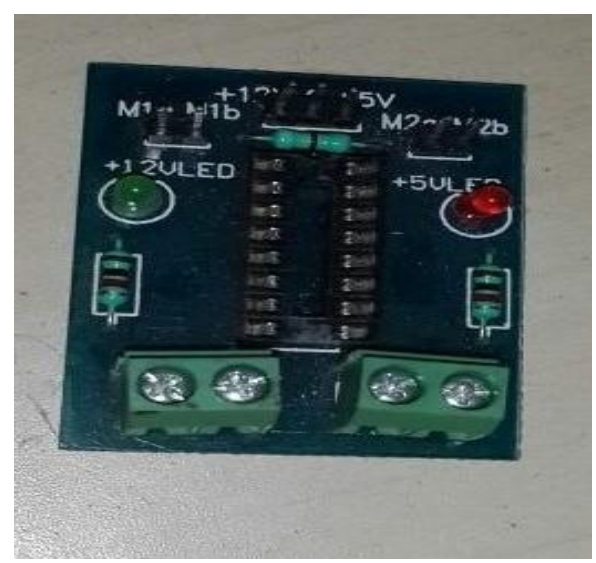

Figure 8: L293D Motor Driver 
a) Sorting of the products according to their colour.

b) Counting of the number of objects passed and differentiated according to their colour.

c) Industrial uses like for manufacturing and material handling.

d) Used in laboratories and workshops.

e) Used in airports, museums and malls.

\section{RESULT}

The final result of the project was quite satisfactory. The colour detecting sensor worked well and it was able to detect red, green and blue colour nicely and the sorting of the object with the help of robotic arm was also done in proper direction. DC motors worked well in both cases of robotic arm and conveyor belt. The movement of the belt was smooth without any conflicts. The LCD display also showed correct colour with its count number and description. The overall system performed well as programmed and detects objects according to their colour.

\section{LIMITATION}

a) Here only three coloured (red, green and blue) objects were able to detect.

b) The speed of the conveyor belt was not set according to its timings leading to slippage of the belt.

c) The movement of the robotic arm was restricted to some extent only.

\section{FURTHER DEVELOPMENT}

a) We can add a load cell for the measurement of the weight of the objects.

b) Speed of the conveyor belt can be increased and set using the timing technique.

c) The DC motors can be replaced by the stepper motors.

d) The system productivity can be enhanced by using different sensors.

e) A pneumatic actuator can be used for sorting and placing of the objects.

\section{CONCLUSION}

Nowadays in highly competitive industrial manufacturing, the management of the integrity of supply of a product from raw material to finished product through quality manufacturing is of very much importance. For the product bearing high quality and dimensional accuracy is mandatory. So this project of automatic color sorting is an excellent one because of its working principle and wide implementation. By applying the idea of this project an industry can easily sort the required product according to its color. Though it has some limitations, but by having done some modification this concept can be implemented in wide range of application.

\section{ACKNOWLEDGEMENT}

We would like to express our gratitude and appreciation to our honorable project guide, Mr. Mahesh Gupta, Assistant Professor, Department of Mechanical Engineering, SRM University for his positive attitude and guidance which was very helpful for the completion of the project. We would also like to express our sincere gratitude to all of our respective teachers for their support. Finally thanks to almighty because of which everything was made to be happened.

\section{REFERENCES}

[1] M. Frank, Nobert Kaiser, Wolfgang Buss, Ramona Eberhardt, "High-speed industrial color and position sensors", Electronic Imaging'99, 50-57, 1999.

[2] D.J. Lee and R. S. Anbalagan, "High-speed automated colorsorting vision system", in Optical Engineering Midwest'95, 573-579, 1995.

[3] Norfazlinda Binti Daud, “Application of colors sensor in an automated system”, TechnicalUniversity Malaysia, May 2007.

[4] Bozma and Yal-cin, "Visual processing and classification of items on a moving conveyor: a selective perception approach", vol.18, issue 2, 2002.

[5] R Mattone, G. Campagiorni, F. Galati, "Sorting of items on a moving conveyor belt. Part1: A Technique for detecting and classifying objects", vol. 16, issues 2-3, 1999.

[6] C.Kunhinmohammed, et al, "Automatic Color Sorting Machine Using TCS230 Color Sensor And PIC Microcontroller", vol. 2, issue 2, 2015, 33-38.

[7] M. Dhanoj, K.V. Reshma, V. Sheeba, P. Marymol, “Colour Sensor Bases Object Sorting Robort Using Embedded System”, vol. 4, issue 4, April 2015, 619-622.

[8] Dharmannagari Vinay Kumar Reddy, "Sorting Of Objects Based On Colour By Pick And Place Robotic Arm And With Conveyor Belt Arrangement", Vol. 3, No. 1, January 2014, 67-74.

[9] 'Fuller, J.L., Robotics: Introduction, Programming and Projects, Prentice Hall, 2nd edition, Columbus, Ohio.

[10] Cutkosky, M.R., and McCormick, P., "End Effecters and Tooling", Chapter 14.4-Robotics, in CRC Handbook of Mechanical Engineering, 14.24-14.32. 